DR. ROSEMARY TOWNSEND (Orcid ID : 0000-0002-3438-7069)

MR. JOHN ALLOTEY (Orcid ID : 0000-0003-4134-6246)

PROF. ALEXANDER HEAZELL (Orcid ID : 0000-0002-4303-7845)

BEN WJ MOL (Orcid ID : 0000-0001-8337-550X)

GORDON SMITH (Orcid ID : 0000-0003-2124-0997)

DR. PETER VON DADELSZEN (Orcid ID : 0000-0003-4136-3070)

Article type : Systematic review

\title{
Prediction of stillbirth: an umbrella review of evaluation of prognostic variables
}

Townsend $\mathrm{R}^{1,2}$, Sileo $\mathrm{FG}^{1,2}$, Allotey $\mathrm{J}^{3,4}$, Dodds $\mathrm{J}^{4,5}$, Heazell $\mathrm{A}^{6,7}$, Jorgensen $\mathrm{L}^{8}$, Kim $\mathrm{V}$. $B^{9}$, Magee $L^{10}$, Mol $B^{11}$, Sandall $J^{12,13}$, Smith $G C S^{14,15}$, Thilaganathan $B^{1,2}$, von Dadelszen $P^{10}$, Thangaratinam $S^{3,4}$, Khalil $A^{1,2}$.

1. Molecular and Clinical Sciences Research Institute, St George's, University of London and St George's University Hospitals NHS Foundation Trust, London, UK

2. Fetal Medicine Unit, St George's University Hospitals NHS Foundation Trust, London, UK

3. Institute of Metabolism and Systems Research, University of Birmingham, Birmingham, UK

This article has been accepted for publication and undergone full peer review but has not been through the copyediting, typesetting, pagination and proofreading process, which may lead to differences between this version and the Version of Record. Please cite this article as doi:

$\underline{10.1111 / 1471-0528.16510}$

This article is protected by copyright. All rights reserved 
4. Pragmatic Clinical Trials Unit, Barts and the London School of Medicine and Dentistry, Queen Mary University of London, London, UK

5. Centre for Women's Health, Institute of Population Health Sciences, Barts and the London School of Medicine and Dentistry, Queen Mary University of London, London, UK

6. St. Mary's Hospital, Manchester Academic Health Science Centre, Manchester University NHS Foundation Trust, Manchester, UK.

7. Maternal and Fetal Health Research Centre, School of Medical Sciences, Faculty of Biology, Medicine and Health, University of Manchester, Manchester, UK.

8. Katie's Team, East London, United Kingdom.

9. The Robinson Institute, University of Adelaide, Adelaide, SA, Australia.

10. School of Life Course Sciences, Faculty of Life Sciences and Medicine, King's College London, London, United Kingdom.

11. Department of Obstetrics and Gynaecology, School of Medicine, Monash University, Melbourne, Australia

12. Centre for Implementation Science, Health Service and Population Research Department, Institute of Psychiatry, Psychology and Neuroscience, King's College London, London, United Kingdom

13. Department of Women and Children's Health, School of Life Course Sciences, Faculty of Life Sciences \& Medicine, King's College London, St. Thomas' Hospital, London, United Kingdom

14. Department of Obstetrics and Gynaecology, University of Cambridge, NIHR Cambridge Biomedical Research Centre, Cambridge, United Kingdom

15. Centre for Trophoblast Research (CTR), Department of Physiology, Development and Neuroscience, University of Cambridge, Cambridge, United Kingdom

Corresponding author: Dr Rosemary Townsend

Molecular and Clinical Sciences Research Institute

St George's University of London

Cranmer Terrace

London

SW17 ORE

This article is protected by copyright. All rights reserved 
Email: Rosemary.townsend1@nhs.net

Phone: 07740285018

Running head: Umbrella review of predictors of stillbirth ABSTRACT

Background: Stillbirth accounts for over 2 million deaths a year worldwide, and rates remains stubbornly high. Multivariable prediction models may be key to individualised monitoring, intervention or early birth in pregnancy to prevent stillbirth.

Objectives: To collate and evaluate systematic reviews of factors associated with stillbirth in order to identify variables relevant to prediction model development.

Search strategy: Medline, Embase, DARE and Cochrane Library databases and reference lists were searched up to November 2019.

Selection criteria: We included systematic reviews of association of individual variables with stillbirth without language restriction.

Data collection and analysis: Abstract screening and data extraction were conducted in duplicate. Methodological quality was assessed using AMSTAR and QUIPS criteria. The evidence supporting association with each variable was graded.

Results: The search identified 1198 citations. 69 systematic reviews reporting 64 variables were included. The most frequently reported were maternal age $(n=5)$, BMI $(n=6)$ and maternal diabetes $(n=5)$. Uterine artery Doppler appeared to have the best performance of any single test for stillbirth. The strongest evidence of association was for nulliparity and pre-existing hypertension.

Conclusion: We have identified variables relevant to the development of prediction models for stillbirth. Age, parity and prior adverse pregnancy outcomes had a more convincing association than the best performing tests which were PAPP-A, PIGF and 
UtAD. The evidence was limited by high heterogeneity and lack of data on intervention bias.

Funding: The project was funded by the Stillbirth and Neonatal Death Society (SANDS) as part of a grant for prediction and prevention of stillbirth and perinatal mortality.

Keywords: Systematic reviews, epidemiology: perinatal, fetal medicine: perinatal diagnosis, ultrasound, fetal medicine: serum screening

Tweetable abstract: Review shows key predictors for use in developing models predicting stillbirth include age, prior pregnancy outcome and PAPP-A, PLGF and Uterine artery Doppler.

This article is protected by copyright. All rights reserved 


\section{INTRODUCTION}

Stillbirth accounts for more global deaths than HIVIAIDS or cancer; (1) although recent years have seen a steady fall in maternal and neonatal mortality, global incidence of stillbirth remains stubbornly high. The majority of the burden occurs in low and middle income settings, but stillbirth reduction is an urgent priority worldwide. The UK incidence of stillbirth fell by a fifth between 1993 and 2015, (2) but remains one of the highest in Europe. ${ }^{(3)}$

On a global scale, stillbirth prevention includes addressing population level issues including malaria and syphilis treatment and optimising nutrition. ${ }^{(4)}$ In the UK, attention is focused on antenatal identification of high risk pregnancies. ${ }^{(5)}$ Consultation with patients and stakeholders has established that developing new antenatal testing strategies to prevent stillbirth is a key research priority. ${ }^{(6)}$

Current national guidelines recommend identifying women with any known risk factors for stillbirth as high risk. (7) In most cases, there has been no formal evaluation of these risk factors as clinical tests or consideration that other factors present may modify the risk of stillbirth.

The most important cause of stillbirth is placental dysfunction, but maternal and fetal comorbidities and environmental and genetic factors also play a significant role. ${ }^{(8)}$ Given this heterogeneity, prediction by single variables is unlikely to be clinically useful. ${ }^{(9)}$ Instead, multivariable prediction models are likely to yield clinically relevant results. (9) Selection of variables for the development of prediction models is often limited by variables commonly available in large datasets ${ }^{(10,11)}$ but optimal model development would take into account all available evidence, including promising new candidate variables. ${ }^{(12)}$

In order to prioritise variables for inclusion in any model for the prediction of stillbirth we must first critically appraise the available evidence. We undertook an umbrella review to collate and evaluate systematic reviews of risk factors for stillbirth with the aim of 
identifying variables that could be relevant to the development of a clinical prediction model for stillbirth.

\section{METHODS}

The systematic review was registered with PROSPERO (Registration number: CRD42017074061)(13-15) and reported according to the PRISMA guidelines. ${ }^{(16)}$ Patients were not directly involved in the design of this review.

\section{Literature search}

We searched Medline, EMBASE and the Cochrane Library from inception to November 2019 using combinations of the relevant medical subject heading (MeSH) terms, key words, and word variants for "stillbirth", "stillborn", "meta-analysis" and "review" without language restrictions. (Appendix S1) Reference lists of relevant articles were handsearched for additional relevant papers.

\section{Study selection and data extraction}

Two reviewers (RT and FGS) reviewed all abstracts independently. Any discrepancies were resolved by consensus. We obtained full texts of reviews that met the inclusion criteria. We included reviews that assessed the predictive accuracy or association of single variables with stillbirth. (Table 1, Table S1a) The steering group (AK, ST, RT and FGS) reviewed the list of variables identified at the full text review stage and excluded those deemed by consensus to be unlikely to contribute to a useful clinical prediction model, including rare co-morbidities and environmental exposures. (Table S1b) We excluded reviews evaluating the association of therapeutic drugs with stillbirth. We excluded genetic association studies, but included common thrombophilia mutations as these may be identified during routine care. Variables exclusively related to stillbirth in LMIC settings (eg malaria) were excluded, but reviews from LMIC settings were included where the variables were generalisable. The contributory factors ${ }^{(17)}$ and available variables in LMIC are so different as to mandate a separate approach to prediction of stillbirth. $(18,19)$

This article is protected by copyright. All rights reserved 
The variables identified were classified as clinical characteristics, biochemical or ultrasound variables. We included reviews of observational and prediction studies evaluating tests in the first, second and third trimester. We accepted and noted the authors' definition of stillbirth. There is no published core outcome set for stillbirth studies and significant variation in outcome reporting was anticipated.

We defined a review as systematic if they included an explicit method for searching the literature, searched $>2$ databases, and provided clear inclusion and exclusion criteria. Case reports, primary studies, narrative reviews and poster abstracts were excluded. Two reviewers (RT, FGS) independently extracted relevant data. We obtained data on publication year, study funding, databases searched, studies included, number of pregnancies/women and number of stillbirths, definition of stillbirth, inclusion/exclusion criteria, variables evaluated, timing of testing and degree of association.

\section{Quality assessment}

Two reviewers (RT, FGS) assessed the methodological quality of the included reviews using the AMSTAR tool (Appendix S2). (20) The tool evaluates whether the reviewers incorporated the following: a prospectively designed study with a clear research question, a comprehensive literature search, relied on the status of publication as an inclusion criterion, duplicated study selection and data extraction, gave details of both included and excluded studies, assessed and documented the risk of bias of the included studies, included information on the funding of primary studies, used appropriate statistical methods to combine the findings of studies and considered the impact of the risk of bias and study heterogeneity in primary studies on the analysis and results, assessed the likelihood of publication bias and reported any conflict of interest.

Because the outcome of interest was the prognostic value of the variables considered, we additionally considered whether the risk of bias in the included studies in each of the key domains identified by the Quality In Prognosis Studies tool (QUIPS) had been assessed. (Appendix S3) The six domains are study participation, attrition, measurement of the predictive variable and the outcome, adjustment for confounders and the quality of analysis and reporting. 
Systematic grading of the evidence of association

The purpose of an umbrella review is to provide a broad overview of the field and to assess the strength of the evidence supporting an association or effect.(21) For each variable we identified the meta-analysis with the most component studies reporting sufficient data for comparison. We considered the sample size to be the number of events, not the number of patients. Evidence of association was considered highly convincing with $>1000$ events, highly statistically significant summary associations $\left(p<10^{-}\right.$ $\left.{ }^{6}\right)$ with no large heterogeneity $\left(I^{2}<50 \%\right)$ and no concern about small study effects. Where the sample size was $>1000$ and $p<10^{-6}$ but there were concerns about heterogeneity or small study effects the grading was reduced to highly suggestive. When the p-value was $<0.001$ the evidence was graded as suggestive, while meta-analysis supported by $<1000$ cases but with a $p$-value $<0.05$ were considered weak evidence of association. Where the $p$-value was not reported in the original meta-analysis this was calculated.(22) In some very large studies the number of events was not reported. Assuming a conservative prevalence of stillbirth of $0.5 \%$, we included studies with an overall sample size $>200000$ as likely to have sufficient events. All calculations were performed in Excel.

\section{RESULTS}

The literature search identified 1198 citations. After screening abstracts, 266 full text papers were retrieved for review, of which 197 were excluded. (Figure 1, Table S1a) Sixty-nine systematic reviews were included. $(3,9,17,23-88)$

\section{Quality assessment using AMSTAR}

The methodological quality of the included systematic reviews was assessed using the AMSTAR checklist (Figure 2, Appendix S2). The mean score was 7.4/11 and 76.8\% $(53 / 69)$ of the included studies had an AMSTAR score greater than or equal to 7 . Fiftyeight studies $(84.0 \%)$ declared conflicts of interest. Nineteen studies $(27.5 \%)$ did not specify funding sources and $13(18.8 \%)$ reported no study funding.

This article is protected by copyright. All rights reserved 
Quality assessment using QUality In Prognosis Studies (QUIPS)

We assessed the risk of bias relating specifically to domains important in prognostic research. (Figure 2) Although most included studies suggested that their findings were relevant to stillbirth prediction, none reported fully on the risk of bias in all QUIPS domains. Most studies (54/69, 78.2\%) considered the definition and representativeness of the participants in the primary studies and the adequacy of definition and assessment of exposure $(57 / 69,82.6 \%)$ and outcome $(55 / 69,79.7 \%)$. Only $44 / 69(63.7 \%)$ noted adjustment for potential confounders or the lack of it in the included studies and just $15 / 69(21.7 \%)$ considered the impact of loss to follow up on the apparent performance of the predictive variables.

\section{Characteristics of the included studies}

The included reviews considered 64 individual variables. (Table 1) The characteristics of the included studies are summarised in Table S2 and Figure 3. The majority of included reviews reported on maternal characteristics such as maternal age, parity, body mass index (BMI), smoking, caffeine and alcohol intake. Medical co-morbidities and past pregnancy outcomes were additionally classified as maternal characteristics. Ultrasound markers reviewed included UtAD, cerebroplacental ratio (CPR), nuchal translucency (NT), echogenic bowel, fetal sex and fetal growth. Biochemical parameters investigated included thrombophilia associated markers (including anticardiolipin antibodies (ACA), lupus anticoagulant (LA) and homocysteine), markers of fetoplacental unit function (human chorionic gonadotrophin (hCG), alpha feto protein (AFP), pregnancy associated plasma protein-A (PAPP-A)) and others including thyroid stimulating hormone (TSH), soluble fms-like tyrosine kinase-1 (sFlt-1), serum uric acid, vitamin D, proteinuria and cell free fetal DNA (cffDNA).

\section{Outcome reporting}

The majority of included reviews $(n=34)$ summarised their findings with odds ratios (OR) (Figure $4 a)$ or via narrative synthesis $(n=16)$, while others reported relative risk (RR, $\mathrm{n}=10$ ) (Figure $4 \mathrm{c}$ ), likelihood ratios $(L R, n=4)$ (Figure $4 b)$ or sensitivity and specificity $(n=3)$. Other estimates of association included effect size, population attributable risk and 
crude incidence. There was also significant variation in outcome reporting both in the reviews and the primary studies. Reported definitions of stillbirth varied in gestational cut offs (range 10-28 weeks) and in the pathology of stillbirth - several excluded congenital anomalies or 'explained' stillbirths.

\section{Maternal characteristics}

The majority of identified reviews and the strongest identified evidence related to maternal characteristics. The most frequently reported were maternal age (particularly age $>35$ years, $n=5)$, BMI or other measures of obesity $(n=6)$ and maternal diabetes $(n=5)$. The association of BMI with stillbirth was supported by highly convincing evidence, and maternal age by highly suggestive evidence. (Table 2) Of the maternal medical conditions reported on, the greatest degree of association was reported with sickle cell disease (1 review, $\mathrm{RR} 3.99,95 \% \mathrm{Cl}$ 2.63-6.04), and the supporting evidence was rated as highly suggestive. The value of this variable in a prediction model would relate to the local incidence of sickle cell in the population of intended use. Prior pregnancy history was also strongly associated with stillbirth; a prior stillbirth (2 reviews, OR of $4.83,95 \% \mathrm{Cl}$ $3.77-6.18),{ }^{(48)}$ a prior preterm birth (1 review, OR 2.98, 95\% Cl 2.05-4.34) and a prior birth of a small-for-gestational-age (SGA) baby before 34 weeks (1 review, OR 6.00, 95\% CI 3.43-10.49)(49) all supported by highly suggestive or suggestive evidence.

Socioeconomic factors ranging from social deprivation to immigration status and education were associated with stillbirth, chiefly supported by lower quality evidence. Ethnicity was considered in two reviews: in one indigenous or aboriginal status was evaluated $^{(59)}$ and in one stillbirth risk was compared between White, Black and Asian

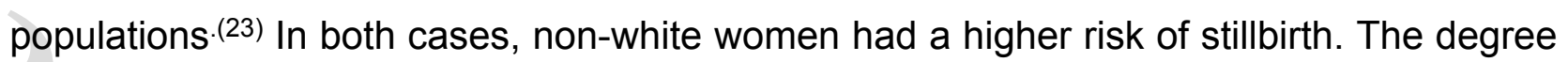
of association was comparable to maternal age, BMI and prior birth of an SGA baby and supported by highly suggestive evidence.

Maternal smoking was consistently associated with risk of stillbirth. Two studies demonstrated a plausible biological gradient of increasing risk with increasing exposure. ${ }^{(63,64)}$ Caffeine and alcohol use were not consistently associated with stillbirth. 


\section{Ultrasound markers}

CPR, assessed in three reviews, appeared to have the best performance of any single variable; sensitivity was reported for perinatal death (stillbirth and neonatal death) as $93 \%$ (95\% Cl $71-99 \%)$ and specificity $74 \%(95 \% \mathrm{Cl} 60-84 \%) .{ }^{(74)}$ For stillbirth specifically, the OR was 3.99 (95\% Cl 1.81-8.8). (73) Second trimester UtAD had a pooled sensitivity of $65 \%(95 \% \mathrm{Cl} 38-85 \%)$ and specificity $82 \%(95 \% \mathrm{Cl} 72-88 \%)$ for stillbirth, with OR 8.3 $(95 \% \mathrm{Cl} 3.0-22.4)^{(71)}$ Similarly, suboptimal fetal growth had a sensitivity of $32 \%(95 \% \mathrm{Cl}$ 31-34) and specificity $75 \%$ (95\% Cl 75-75\%) for stillbirth. ${ }^{(9)}$ Other markers associated with stillbirth included NT, echogenic bowel and male sex. ${ }^{(9)}$ Reviews of ultrasound measures including CPR, UtAD and MCA were limited by variation in the definition of an abnormal result and by small sample sizes. The association was consistent, but the supporting evidence is still weak at best.

\section{Biochemical markers}

Key biochemical tests include AFP (two reviews) [AFP>2.0 MoM; Sens 11\% (95\% Cl 913\%) Spec: $96 \%(95 \% \mathrm{Cl} 96-96 \%))^{(9)}$ and PAPP-A (two reviews) [PAPP-A <0.4 MoM; Sens. $15 \%(95 \%$ Cl 8-26\%) Spec $95 \% \quad(95 \% \mathrm{Cl}$ 95-96)).(9) Human chorionic gonadotrophin (hCG) (two reviews) had sensitivity of $4 \%$ (95\% Cl 1-14\%) and specificity 94\% (95\% Cl 93-94\%) in one review, (9) but the other found that hCG added little value to AFP in combination. ${ }^{(84)}$ Placental growth factor (PIGF) is associated with placental function and was reported with a large degree of association, with a diagnostic OR of $49.2(95 \% \mathrm{Cl} 12.7-191)$ in two reviews. ${ }^{(78,82)}$

Several thrombophilia markers showed a strong association with stillbirth including LA (two studies, OR 4.3-54.18) $(77,89)$ and ACA (two studies, OR 4.29-15.17).(77) The Factor $\mathrm{V}$ Leiden mutation, protein $\mathrm{S}$ deficiency and activated protein $\mathrm{C}$ resistance (APCR) were all also strongly associated with stillbirth with OR 6.11 (95\% Cl 2.8-13.2), 16.2 (95\% Cl 5.1-52.3) and 5.0 (95\% Cl 2.0-12.4), respectively. ${ }^{(77)}$

All of these studies were limited by high levels of heterogeneity and small sample sizes.

\section{Grading of evidence}


Three variables were supported by highly convincing evidence - nulliparity, pre-existing hypertension and increased maternal BMI. (Table 2) A further seven variables were supported by highly suggestive evidence: maternal age, fetal sex, a history of SGA, ethnicity, sickle cell disease, cigarette smoking and aboriginal or indigenous status. Notably, the strongest evidence available was for elements of maternal medical history and simple physical examination. All biomarker and ultrasound variables were supported by weak evidence at best, (Tables S3 and S4) although the limited evidence available did support significant associations, particularly for PLGF, CPR and uterine artery Doppler.

\section{DISCUSSION}

Main findings

This review has identified 69 systematic reviews examining 64 variables potentially associated with stillbirth. No marker had useful screening performance, but several were consistently and strongly associated with stillbirth. Importantly, isolated factors from the obstetric history and examination including age, BMI and prior adverse pregnancy outcomes were better supported by the available evidence than even the most strongly associated tests which were PAPP-A, PIGF, CPR and second trimester UtAD.

\section{Strengths and limitations}

Strengths of this review include the comprehensive search and critical evaluation in synthesising a massive quantity of literature. The study was limited by the quality of included reviews, notably in relation to factors important to prediction.

There was substantial missing information relating to measurement of exposures and outcomes and significant variation in outcome reporting was noted. There was variation in the quantities used to assess association - most commonly OR or RR, limiting direct comparisons and lacking information on sensitivity. The variation in the outcomes reported limited comparisons between studies. Abou Nasser et al reported significant associations between anti-phospholipid antibodies and stillbirth, ${ }^{(89)}$ but used the outcome of fetal loss $>10$ WGA excluding congenital anomaly which may have led to over estimation of the association. In sub-group analysis, the association with fetal loss $>20$ WGA was less convincing. Similarly, CPR and UtAD performed well as single tests but in 
two reviews of CPR sensitivity for perinatal death was reported without the corresponding sensitivity for stillbirth alone, ${ }^{(74,85)}$ limiting direct comparison with UtAD.

The competing risks of stillbirth or livebirth may negatively affect observed predictive accuracy of tests, but were not considered in included reviews. Where a high risk of stillbirth is identified but birth occurs before stillbirth, the case will seem to be a false positive. This is particularly significant for 'late' stillbirths, since it is increasingly likely that birth will supervene and consistent with the observation that tests for predicting early stillbirth are more accurate than those predicting later stillbirth. (9)

Arguably, early birth is most likely to occur in those at highest risk because clinicians act on risk factors for stillbirth. Only three reviews considered this risk of bias and of these, the risk was low in the reviews assessing biochemical markers ${ }^{(84)}$ and Doppler ${ }^{(71)}$ but increased in the review including clinical characteristics. ${ }^{(9)}$

The strength of evidence is related to the sample size for assessment and when considering a rare event like stillbirth, large sample sizes are required to convincingly support a statistical association. In the case of novel biomarkers or ultrasound testing outwith routine care, the costs of assembling such large samples are restrictive. As work continues on evaluation of promising tests, the future may see better evidence supporting variables such as PLGF, CPR and PAPP-A.

\section{Interpretation}

Previous reviews of individual predictors of stillbirth have concluded that multivariable models are likely to be required for meaningful clinical impact. ${ }^{(3,9)}$ In this review we have systematically evaluated factors associated with stillbirth in order to identify variables most relevant to the development of such models. It would not be appropriate to suggest that the strength of association between a single variable and stillbirth would necessarily predict the value of that variable as a predictor in a model. This information is simply presented to inform model developers identifying candidate predictors for model development and should be considered in the light of the intended population and clinical application. 
Although clinical characteristics were the variables most convincingly associated with stillbirth in this review, only $19 \%$ of stillbirths are associated with established clinical risk factors, ${ }^{(90)}$ and triage based on these alone has a poor PPV. ${ }^{(19)}$ The three variables most consistently associated with stillbirth (nulliparity, pre-existing hypertension and increased maternal BMI) clearly relate to maternal and placental vascular function and are included in national guidelines as recognised risk factors for placental dysfunction, ${ }^{(5)}$ as are maternal age, previous SGA and cigarette smoking. Three promising prognostic tests include PLGF, UtAD and CPR, all of which also primarily relate to placental dysfunction.

A recent systematic review of prediction models in obstetrics found three models for stillbirth. (91) These models included UtAD and ethnicity with history of prior pregnancy loss in one and with BMI in the second, all variables identified as important predictors in this review. ${ }^{(92)}$ Further models have subsequently been developed (18,93,94) but none yet externally validated. Although increasing interest in individualising care has led to increasing numbers of models, transfer to clinical practice has been hampered by a lack of subsequent external validation and clinical evaluation.(95)

Socioeconomic deprivation was consistently associated with stillbirth in both high and low income settings but is measured and defined heterogeneously, limiting the utility of this variable. Similarly, the association of ethnicity with adverse pregnancy outcomes is supported by strong statistical evidence, but problematic as a predictive variable. Selfreported ethnicity varies from clinician perceived ethnicity and rapidly loses specificity in diverse populations with high proportions of 'mixed' ethnicities. ${ }^{(96)}$ Although the observed association is potentially related to biological factors (length of pregnancy and cardiovascular parameters differ with ethnicity and are plausibly associated with stillbirth), it is undoubtedly confounded by factors like higher multiparity in selected groups, structural racism and systemic inequality in access to healthcare. In the light of the consistent findings of the MBRRACE reports that perinatal mortality disproportionately affects Afro-Caribbean babies in the UK, addressing ethnicity based inequity in healthcare is of prime importance, but ethnicity or socioeconomic status as a predictive variable may limit any model developed to only that population in which it is developed. 
Nonetheless, these findings reinforce the importance of addressing social inequality as a core strategy for the prevention of stillbirth in any setting.

\section{Conclusion}

Clinical and research implications

Informal screening to identify high risk pregnancies is embedded in practice and urgently needs to be improved. Development of robust models remains a challenge because of the rarity of stillbirth as an outcome. The heterogeneous causes of stillbirth may be best addressed by separate models; logically, the initial target should be placental dysfunction, representing the largest and most clearly defined factor contributing to stillbirth. Separate models by gestation could also allow continuous risk assessment through pregnancy, taking into account recently available patient data.

Model development requires a large volume of data with detailed information on a number of candidate predictors and can be optimised by maximising available data and minimising candidate predictors in order to arrive at the best achievable effective sample size.(97) In this review we have identified several key candidate variables which should be considered in model development; maternal age, BMI, parity, essential hypertension, diabetes, history of previous stillbirth, cigarette smoking, uterine artery Doppler, PAPP-A and PIGF.

A large-scale, collaborative approach utilising individual participant data (IPD) metaanalysis offers an innovative approach to addressing the problems of stillbirth prediction. IPD meta-analysis allows the use of all original data and continuous variables with the flexibility to standardise variable and outcome definitions, their combinations and comparisons across datasets.(98) Existing models could be validated and tested against new models,(99) offering the opportunity to build consensus around development and validation of methodologically robust models.

In this era of increasingly personalised medicine, women want individualised recommendations for care and expect clinicians to make the most effective use of available tests. The global loss of millions of lives to stillbirth every year is too significant 
a tragedy to waste time generating excessive clinically irrelevant prediction models; the time has come to initiate a collaborative approach in order to definitively answer the question of how to predict, and ultimately prevent, stillbirth.

\section{Acknowledgements}

The authors would like to acknowledge collaborators in the International Prediction of Pregnancy Complications group and Mehali Patel, of the Stillbirth and Neonatal Death Society. (SANDS)

\section{Disclosure of interests}

Ben Mol (BWM) reports consultancy for ObsEva, Merck and Guerbet, Shakila Thangaratinam (ST) is the $\mathrm{Cl}$ and Asma Khalil (AK) and Basky Thilaganathan (BT) are co-PI of the NIHR funded IPD meta-analysis IPPIC to predict pregnancy complications. Jane Sandall is an NIHR Senior Investigator and is also supported by the National Institute for Health Research (NIHR) Applied Research Collaboration South London (NIHR ARC South London) at King's College Hospital NHS Foundation Trust. The views expressed are those of the author[s] and not necessarily those of the NIHR or the Department of Health and Social Care. The remaining authors have no disclosures. Completed disclosure of interest forms are available to view online as supporting information.

\section{Contribution to authorship}

RT planned and carried out the search, data extraction and analysis and drafted the manuscript. FGS carried out abstract screening, data extraction and reviewed and edited the manuscript. JA contributed to data analysis and reviewed and edited the manuscript. JD, AH, LJ, BK, LM, BWM, JS, GS, BT and PVD all contributed to the design of the study and reviewed and edited the manuscript. ST and AK conceived of and directed the design of the study, chaired the steering group and reviewed and edited the manuscript.

\section{Details of ethics approval}

No ethics approval was required for this systematic review in keeping with the National Research Ethics Service assessment. 
Funding: The project was funded by the Stillbirth and Neonatal Death Society (SANDS) as part of a grant for prediction and prevention of stillbirth and perinatal mortality.

This article is protected by copyright. All rights reserved 


\section{REFERENCES}

1. Blencowe H, Cousens S, Jassir FB, Say L, Chou D, Mathers C, et al. National, regional, and worldwide estimates of stillbirth rates in 2015, with trends from 2000: a systematic analysis. Lancet Glob Heal. 2016 Feb 1;4(2):e98-108.

2. ONS. Childhood mortality in England and Wales - Office for National Statistics. 2017.

3. Flenady V, Koopmans L, Middleton P, Frøen JF, Smith GC, Gibbons K, et al. Major risk factors for stillbirth in high-income countries: a systematic review and metaanalysis. Lancet (London, England). 2011;377(9774):1331-40.

4. Bhutta ZA, Darmstadt GL, Haws RA, Yakoob MY, Lawn JE. Delivering interventions to reduce the global burden of stillbirths: improving service supply and community demand. BMC Pregnancy Childbirth. 2009 May 7;9 Suppl 1(Suppl 1):S7-S7.

5. NHS England. Saving Babies' Lives A care bundle for reducing stillbirth. NHS Engl. 2016;

6. Heazell AEP, Whitworth MK, Whitcombe J, Glover SW, Bevan C, Brewin J, et al. Research priorities for stillbirth: process overview and results from UK Stillbirth Priority Setting Partnership. Ultrasound Obstet Gynecol. 2015 Dec;46(6):641-7.

7. The Investigation and Management of the Small-for-Gestational-Age Fetus: Greentop Guideline 31. London, UK; 2013.

8. Gardosi J, Kady SM, McGeown P, Francis A, Tonks A. Classification of stillbirth by relevant condition at death (ReCoDe): population based cohort study. BMJ. 2005 Nov 12;331(7525):1113-7.

9. Conde-Agudelo A, Bird S, Kennedy SH, Villar J, Papageorghiou AT. First- and second-trimester tests to predict stillbirth in unselected pregnant women: a systematic review and meta-analysis. BJOG. 2015;122(1):41-55.

10. Akolekar R, Tokunaka M, Ortega N, Syngelaki A, Nicolaides KH. Prediction of stillbirth from maternal factors, fetal biometry and uterine artery Doppler at 19-24 weeks. Ultrasound Obstet Gynecol. 2016;48(5):624-30.

11. An JJ, Ji HY, You JY, Woo SY, Choi SJ, Oh S young, et al. Introduction of a nomogram for predicting adverse pregnancy outcomes based on maternal serum markers in the quad screen test. Arch Gynecol Obstet. 2015;292(3):589-94. 
12. Steyerberg EW. Clinical Prediction Models. New York, NY: Springer New York; 2009. (Statistics for Biology and Health).

13. HENDERSON LK, CRAIG JC, WILLIS NS, TOVEY D, WEBSTER AC. How to write a Cochrane systematic review. Nephrology. 2010 Sep;15(6):617-24.

14. NHS Centre for Reviews and Dissemination. Systematic reviews: CRD's guidance for undertaking reviews in health care. York; 2009.

15. von Elm E, Altman DG, Egger M, Pocock SJ, Gøtzsche PC, Vandenbroucke JP, et al. The Strengthening the Reporting of Observational Studies in Epidemiology (STROBE) statement: guidelines for reporting observational studies. Lancet. 2007 Oct 20;370(9596):1453-7.

16. Liberati A, Altman DG, Tetzlaff J, Mulrow C, Gøtzsche PC, loannidis JPA, et al. The PRISMA statement for reporting systematic reviews and meta-analyses of studies that evaluate healthcare interventions: explanation and elaboration. BMJ. 2009;339.

17. Aminu M, Unkels R, Mdegela M, Utz B, Adaji S, van den Broek N. Causes of and factors associated with stillbirth in low- and middle-income countries: a systematic literature review. BJOG. 2014;121 Suppl:141-53.

18. Kayode GA, Grobbee DE, Amoakoh-Coleman M, Adeleke IT, Ansah E, de Groot $\mathrm{JAH}$, et al. Predicting stillbirth in a low resource setting. BMC Pregnancy Childbirth. 2016;16(1):1-8.

19. Haws RA, Yakoob M, Soomro T, Menezes E V, Darmstadt GL, Bhutta ZA. Reducing stillbirths: screening and monitoring during pregnancy and labour. BMC Pregnancy Childbirth. 2009 May 7;9(Suppl 1):S5.

20. Shea BJ, Bouter LM, Peterson J, Boers M, Andersson N, Ortiz Z, et al. External Validation of a Measurement Tool to Assess Systematic Reviews (AMSTAR). Gagnier J, editor. PLoS One. 2007 Dec 26;2(12):e1350.

21. Bellou V, Belbasis L, Tzoulaki I, Evangelou E, loannidis JPA. Environmental risk factors and Parkinson's disease: An umbrella review of meta-analyses.

Parkinsonism Relat Disord. 2016 Feb;23:1-9.

22. Altman DG, Bland JM. How to obtain the $P$ value from a confidence interval. BMJ. 2011 Aug 8;343:d2304.

23. Muglu J, Rather H, Arroyo-Manzano D, Bhattacharya S, Balchin I, Khalil A, et al. 
Risks of stillbirth and neonatal death with advancing gestation at term: A systematic review and meta-analysis of cohort studies of 15 million pregnancies. Smith GC, editor. PLOS Med. 2019 Jul 2;16(7):e1002838.

24. Amegah AK, Klevor MK, Wagner CL. Maternal vitamin D insufficiency and risk of adverse pregnancy and birth outcomes: A systematic review and meta-analysis of longitudinal studies. PLoS One. 2017;12(3):e0173605.

25. Murphy VE, Wang G, Namazy JA, Powell H, Gibson PG, Chambers C, et al. The risk of congenital malformations, perinatal mortality and neonatal hospitalisation among pregnant women with asthma: a systematic review and meta-analysis. BJOG. 2013;120(7):812-22.

26. Nevis IF, Reitsma A, Dominic A, McDonald S, Thabane L, Akl EA, et al. Pregnancy outcomes in women with chronic kidney disease: A systematic review. Clin J Am Soc Nephrol. 2011;6(11):2587-98.

27. Oteng-Ntim E, Meeks D, Seed PT, Webster L, Howard J, Doyle P, et al. Adverse maternal and perinatal outcomes in pregnant women with sickle cell disease: Systematic review and meta-analysis. Blood. 2015;125(21):3316-25.

28. Rusner $M$, Berg M, Begley C. Bipolar disorder in pregnancy and childbirth: $A$ systematic review of outcomes. BMC Pregnancy Childbirth. 2016;16(1).

29. Upala S, Yong WC, Sanguankeo A. Association between primary Sjögren's syndrome and pregnancy complications: a systematic review and meta-analysis. Clin Rheumatol. 2016;35(8):1949-55.

30. Webb R, Abel K, Pickles A, Appleby L. Mortality in offspring of parents with psychotic disorders: A critical review and meta-analysis. Am J Psychiatry. 2005;162(6):1045-56.

31. Wang Z, Kanguru L, Hussein J, Fitzmaurice A, Ritchie K. Incidence of adverse outcomes associated with gestational diabetes mellitus in low- and middle-income countries. Int J Gynecol Obstet. 2013;121(1):14-9.

32. Wu J, Ma J, Zhang WH, Di W. Management and outcomes of pregnancy with or without lupus nephritis: A systematic review and meta-analysis. Ther Clin Risk Manag. 2018;14:885-901.

33. Glavind MT, Møllgaard MV, Iversen ML, Arendt LH, Forman A. Obstetrical outcome in women with endometriosis including spontaneous hemoperitoneum and bowel 
perforation: a systematic review. Best Pract Res Clin Obstet Gynaecol. 2018;51:41-52.

34. Brown NT, Turner JM, Kumar S. The intrapartum and perinatal risks of sleepdisordered breathing in pregnancy: a systematic review and metaanalysis. Am J Obstet Gynecol. 2018;219(2):147-161.e1.

35. Warland J, Dorrian J, Morrison JL, O’Brien LM. Maternal sleep during pregnancy and poor fetal outcomes: A scoping review of the literature with meta-analysis. Sleep Med Rev. 2018;41:197-219.

36. Zhao J, Xu B, Zhang Q, Li YP. Which one has a better obstetric and perinatal outcome in singleton pregnancy, IVF/ICSI or FET?: A systematic review and metaanalysis. Reprod Biol Endocrinol. 2016;14(1).

37. Cavoretto P, Candiani M, Giorgione V, Inversetti A, Abu-Saba MM, Tiberio F, et al. Risk of spontaneous preterm birth in singleton pregnancies conceived after IVF/ICSI treatment: meta-analysis of cohort studies. Vol. 51, Ultrasound in Obstetrics and Gynecology. 2018. 43-53 p.

38. Balsells M, García-Patterson A, Gich I, Corcoy R. Maternal and fetal outcome in women with type 2 versus type 1 diabetes mellitus: A systematic review and metaanalysis. J Clin Endocrinol Metab. 2009;94(11):4284-91.

39. Gizzo S, Patrelli TS, Rossanese M, Noventa M, Berretta R, Di Gangi S, et al. An update on diabetic women obstetrical outcomes linked to preconception and pregnancy glycemic profile: A systematic literature review. Sci World J. 2013;2013.

40. Yu L, Zeng X-L, Cheng M-L, Yang G-Z, Wang B, Xiao Z-W, et al. Quantitative assessment of the effect of pre-gestational diabetes and risk of adverse maternal, perinatal and neonatal outcomes. Oncotarget. 2017;

41. Ananth C V, Savitz DA. Vaginal bleeding and adverse reproductive outcomes: $A$ meta-analysis. Paediatr Perinat Epidemiol. 1994;8(1):62-78.

42. Downes KL, Grantz KL, Shenassa ED. Maternal, Labor, Delivery, and Perinatal Outcomes Associated with Placental Abruption: A Systematic Review. Am J Perinatol. 2017;34(10):935-57.

43. Berhan $\mathrm{Y}$, Berhan A. A meta-analysis of selected maternal and fetal factors for perinatal mortality. Ethiop J Health Sci. 2014;24 Suppl:55-68.

44. Bradford B, Thompson JM, Heazell A, McCowan LM, McKinlay C. Understanding 
the associations and significance of fetal movements in overweight or obese pregnant women: a systematic review. Acta Obstet Gynecol Scand. 2018;97(1):1324.

45. Liu Y, Ma X, Zheng J, Liu X, Yan T. Pregnancy outcomes in patients with acute kidney injury during pregnancy: A systematic review and meta-analysis. BMC Pregnancy Childbirth. 2017;17(1):1-9.

46. Mohan M, Antonios A, Konje J, Lindow S, Ahmed Syed M, Akobeng A. Stillbirth and associated perinatal outcomes in obstetric cholestasis: a systematic review and meta-analysis of observational studies. Eur J Obstet Gynecol Reprod Biol X. 2019;3:100026.

47. Ovadia C, Seed PT, Sklavounos A, Geenes V, Di Illio C, Chambers J, et al. Association of adverse perinatal outcomes of intrahepatic cholestasis of pregnancy with biochemical markers: results of aggregate and individual patient data metaanalyses. Lancet. 2019;393(10174):899-909.

48. Lamont K, Scott NW, Jones GT, Bhattacharya S. Risk of recurrent stillbirth: systematic review and meta-analysis. BMJ. 2015;350:h3080.

49. Malacova E, Regan A, Nassar N, Raynes-Greenow C, Leonard H, Srinivasjois R, et al. Risk of stillbirth, preterm delivery and fetal growth restriction following exposure in previous birth: systematic review and meta-analysis. Br J Obstet Gynaecol. 2018;125(2):183-92.

50. Moraitis AA, Oliver-Williams C, Wood AM, Fleming M, Pell JP, Smith GCS. Previous Cesarean Delivery and the Risk of Unexplained Stillbirth: Retrospective Cohort Study and Meta-analysis. Obstet Gynecol Surv. 2016;71(1):5-6.

51. O’Neill SM, Kearney PM, Kenny LC, Khashan AS, Henriksen TB, Lutomski JE, et al. Caesarean Delivery and Subsequent Stillbirth or Miscarriage: Systematic Review and Meta-Analysis. PLoS One. 2013;8(1).

52. Keag OE, Norman JE, Stock SJ. Long-term risks and benefits associated with cesarean delivery for mother, baby, and subsequent pregnancies: Systematic review and meta-analysis. PLoS Med. 2018;15(1):1-22.

53. Aune D, Saugstad OD, Henriksen T, Tonstad S. Maternal body mass index and the risk of fetal death, stillbirth, and infant death: a systematic review and metaanalysis. JAMA. 2014;311(15):1536-46. 
54. Gibbs CM, Wendt A, Peters S, Hogue CJ. The impact of early age at first childbirth on maternal and infant health. Paediatr Perinat Epidemiol. 2012;26(SUPPL.

1):259-84.

55. Chu SY, Kim SY, Lau J, Schmid CH, Dietz PM, Callaghan WM, et al. Maternal obesity and risk of stillbirth: a metaanalysis. Am J Obstet Gynecol. 2007;197(3):223-8.

56. Liu P, Xu L, Wang Y, Zhang Y, Du Y, Sun Y, et al. Association between perinatal outcomes and maternal pre-pregnancy body mass index. Obes Rev. 2016;17(11):1091-102.

57. Marchi J, Berg M, Dencker A, Olander EK, Begley C. Risks associated with obesity in pregnancy, for the mother and baby: A systematic review of reviews. Obes Rev. 2015;16(8):621-38.

58. Slack E, Rankin J, Jones D, Heslehurst N. Effects of maternal anthropometrics on pregnancy outcomes in South Asian women: a systematic review. Obes Rev. 2018;19(4):485-500.

59. Shah PS, Zao J, Al-Wassia H, Shah V. Pregnancy and Neonatal Outcomes of Aboriginal Women: A Systematic Review and Meta-Analysis. Women's Heal Issues. 2011;21(1):28-39.

60. Vos AA, Posthumus AG, Bonsel GJ, Steegers EAP, Denktaş S. Deprived neighborhoods and adverse perinatal outcome: a systematic review and metaanalysis. Acta Obstet Gynecol Scand. 2014;93(8):727-40.

61. Weightman AL, Morgan HE, Shepherd MA, Kitcher H, Roberts C, Dunstan FD. Social inequality and infant health in the UK: systematic review and meta-analyses. BMJ Open. 2012;2(3).

62. Leonardi-Bee J, Britton J, Venn A. Secondhand smoke and adverse fetal outcomes in nonsmoking pregnant women: A meta-analysis. Pediatrics. 2011;127(4):734-41.

63. Marufu TC, Ahankari A, Coleman T, Lewis S. Maternal smoking and the risk of still birth: systematic review and meta-analysis. BMC Public Health. 2015;15:239.

64. Pineles BL, Hsu S, Park E, Samet JM. Systematic review and meta-analyses of perinatal death and maternal exposure to tobacco smoke during pregnancy. Am J Epidemiol. 2016;184(2):87-97.

65. Carolan M, Frankowska D. Advanced maternal age and adverse perinatal 
outcome: a review of the evidence. Midwifery. 2011;27(6):793-801.

66. Greenwood DC, Thatcher NJ, Ye J, Garrard L, Keogh G, King LG, et al. Caffeine intake during pregnancy and adverse birth outcomes: a systematic review and dose-response meta-analysis. Eur J Epidemiol. 2014;29(10):725-34.

67. Wikoff D, Welsh BT, Henderson R, Brorby GP, Britt J, Myers E, et al. Systematic review of the potential adverse effects of caffeine consumption in healthy adults, pregnant women, adolescents, and children. Food Chem Toxicol. 2017;109:585648.

68. Henderson J, Gray R, Brocklehurst P. Systematic review of effects of low-moderate prenatal alcohol exposure on pregnancy outcome. BJOG. 2007;114(3):243-52.

69. Inamdar AS, Croucher RE, Chokhandre MK, Mashyakhy MH, Marinho VCC. Maternal Smokeless Tobacco Use in Pregnancy and Adverse Health Outcomes in Newborns: A Systematic Review. Nicotine Tob Res. 2015;17(9):1058-66.

70. Di Mario S, Say L, Lincetto O. Risk factors for stillbirth in developing countries: a systematic review of the literature. Sex Transm Dis. 2007;34(7 Suppl):S11-21.

71. Allen RE, Morlando M, Thilaganathan B, Zamora J, Khan KS, Thangaratinam S, et al. Predictive accuracy of second-trimester uterine artery Doppler indices for stillbirth: a systematic review and meta-analysis. Ultrasound Obstet Gynecol. 2016;47(1):22-7.

72. Mondal D, Galloway TS, Bailey TC, Mathews F. Elevated risk of stillbirth in males: systematic review and meta-analysis of more than 30 million births. BMC Med. 2014;12:220.

73. Moreta D, Vo S, Eslick GD, Benzie R. Re-evaluating the role of cerebroplacental ratio in predicting adverse perinatal outcome. Eur J Obstet Gynecol Reprod Biol. 2019;242:17-28.

74. Vollgraff Heidweiller-Schreurs CA, De Boer MA, Heymans MW, Schoonmade LJ, Bossuyt PMM, Mol BWJ, et al. Prognostic accuracy of cerebroplacental ratio and middle cerebral artery Doppler for adverse perinatal outcome: systematic review and meta-analysis. Ultrasound Obstet Gynecol. 2018;51(3):313-22.

75. Abou-Nassar K, Carrier M, Ramsay T, Rodger MA. The association between antiphospholipid antibodies and placenta mediated complications: A systematic review and meta-analysis. Thromb Res. 2011;128(1):77-85. 
76. Huang L, Sauve R, Birkett N, Fergusson D, Van Walraven C. Maternal age and risk of stillbirth: A systematic review. CMAJ. 2008;178(2):165-72.

77. Alfirevic Z, Roberts D, Martlew V. How strong is the association between maternal thrombophilia and adverse pregnancy outcome? A systematic review. Eur J Obstet Gynecol Reprod Biol. 2002;101(1):6-14.

78. Heazell AE, Hayes DJ, Whitworth M, Takwoingi Y, Bayliss SE, Davenport C. Biochemical tests of placental function versus ultrasound assessment of fetal size for stillbirth and small-for-gestational-age infants. Cochrane Database Syst Rev. 2019 May 14;(5).

79. Jung J, Rahman MM, Rahman MS, Swe KT, Islam MR, Rahman MO, et al. Effects of hemoglobin levels during pregnancy on adverse maternal and infant outcomes: a systematic review and meta-analysis. Ann N Y Acad Sci. 2019;1450:69-82.

80. Young MF, Oaks BM, Tandon S, Martorell R, Dewey KG, Wendt AS. Maternal hemoglobin concentrations across pregnancy and maternal and child health: a systematic review and meta-analysis. Ann N Y Acad Sci. 2019;1450:47-68.

81. Thangaratinam S, Ismail KMK, Sharp S, Coomarasamy A, Khan KS. Accuracy of serum uric acid in predicting complications of pre-eclampsia: A systematic review. BJOG An Int J Obstet Gynaecol. 2006;113(4):369-78.

82. Sherrell H, Dunn L, Clifton V, Kumar S. Systematic review of maternal Placental Growth Factor levels in late pregnancy as a predictor of adverse intrapartum and perinatal outcomes. Eur J Obstet Gynecol Reprod Biol. 2018;225:26-34.

83. Thangaratinam S, Coomarasamy A, O'Mahony F, Sharp S, Zamora J, Khan KS, et al. Estimation of proteinuria as a predictor of complications of pre-eclampsia: $A$ systematic review. BMC Med. 2009;7(10).

84. Hui D, Okun N, Murphy K, Kingdom J, Uleryk E, Shah PS. Combinations of maternal serum markers to predict preeclampsia, small for gestational age, and stillbirth: a systematic review. J Obstet Gynaecol Can. 2012;34(2):142-53.

85. Conde-Agudelo A, Villar J, Kennedy SH, Papageorghiou AT. Predictive accuracy of cerebroplacental ratio for adverse perinatal and neurodevelopmental outcomes in suspected fetal growth restriction: systematic review and meta-analysis. Ultrasound Obstet Gynecol. 2018;

86. Lean SC, Derricott H, Jones RL, Heazell AEP. Advanced maternal age and 
adverse pregnancy outcomes: A systematic review and meta-analysis. PLoS One. 2017;12(10):1-15.

87. Berhan $\mathrm{Y}$, Berhan A. A meta-analysis of socio-demographic factors for perinatal mortality in developing countries: a subgroup analysis of the national surveys and small scale studies. Ethiop J Health Sci. 2014;24:41-54.

88. Oldereid NB, Wennerholm U-B, Pinborg A, Loft A, Laivuori H, Petzold M, et al. The effect of paternal factors on perinatal and paediatric outcomes: a systematic review and meta-analysis. Hum Reprod Update. 2018;(February):1-70.

89. Abou-nassar K, Carrier M, Ramsay T, Rodger MA. The association between antiphospholipid antibodies and placenta mediated complications : A systematic review and meta-analysis. Thromb Res. 2011;128(1):77-85.

90. Group TSCRNW. Association Between Stillbirth and Risk Factors Known at Pregnancy Confirmation. JAMA. 2011 Dec 14;306(22):2469-79.

91. Kleinrouweler CE, Cheong-See F, Collins G, Kwee A, Thangaratinam S, Khan KS, et al. Prognostic models in obstetrics: available, but far from applicable. Am J Obstet Gynecol. 2016;214(1):79-90.

92. S Smith GC, H Yu CK, Papageorghiou AT, Maria Cacho A, Nicolaides KH. Maternal Uterine Artery Doppler Flow Velocimetry and the Risk of Stillbirth LEVEL OF EVIDENCE: II. Vol. 109, Obstet Gynecol. 2007.

93. Trudell AS, Tuuli MG, Colditz GA, Macones GA, Odibo AO. A stillbirth calculator: Development \& internal validation of a clinical prediction model to quantify stillbirth risk. PLoS One. 2017;12(3):1-13.

94. Iliodromiti S, Mackay DF, Smith GCS, Pell JP, Sattar N, Lawlor DA, et al. Customised and Noncustomised Birth Weight Centiles and Prediction of Stillbirth and Infant Mortality and Morbidity: A Cohort Study of 979,912 Term Singleton Pregnancies in Scotland. Myers JE, editor. PLOS Med. 2017 Jan 31;14(1):e1002228.

95. Collins GS, Reitsma JB, Altman DG, Moons K. Transparent reporting of a multivariable prediction model for individual prognosis or diagnosis (TRIPOD): the TRIPOD Statement. BMC Med. 2015 Jan 6;13(1):1.

96. Lockie E, Mccarthy EA, Hui L, Churilov L. Feasibility of using self-reported ethnicity in pregnancy according to the gestation-related optimal weight classification : a 
cross-sectional study. BJOG. 2018;125:704-9.

97. Riley RD, Snell KIE, Ensor J, Burke DL, Harrell FE, Moons KGM, et al. Minimum sample size for developing a multivariable prediction model: Part I - Continuous outcomes. Stat Med. 2018 Oct 22;

98. Abo-Zaid G, Sauerbrei W, Riley RD. Individual participant data meta-analysis of prognostic factor studies: state of the art? BMC Med Res Methodol. 2012 Dec 24;12(1):56.

99. Khan KS, Bachmann LM, ter Riet G. Systematic reviews with individual patient data meta-analysis to evaluate diagnostic tests. Eur J Obstet Gynecol Reprod Biol. 2003 Jun 10;108(2):121-5.

This article is protected by copyright. All rights reserved 
Figure and Table Legends

Figure 1. PRISMA flow chart

Figure 2a. Methodological quality of included reviews

Figure $2 b$. Risk of bias relating to QUIPS domains

Figure 3. Summary of characteristics of included studies

Figure 4. Association of single variables with stillbirth in studies reporting
a) Odds ratios
b) Likelihood ratios
c) Risk ratios

Table 1: List of variables included in this review

Table 2: Variables supported by highly convincing or suggestive evidence

Online Supporting Material

Table S1a: Excluded as did not meet inclusion criteria

Table S1b: Excluded by consensus as not relevant to the development of prediction models

Table S2: Characteristics of included studies

Table S3: Variables supported by weak evidence

Table S4: Variables with no evidence of association

Appendix S1: Literature search strategy

Appendix S2. AMSTAR checklist

Appendix S3. QUIPS checklist 
Table 1. Prognostic variables for stillbirth investigated in the included systematic reviews.

\section{Parental characteristics and history}

Maternal and paternal age

\section{Parity}

Body mass index

Pre-existing medical conditions (epilepsy, vitamin D deficiency, hypertension, asthma, chronic kidney disease, sickle cell disease, bipolar disorder, Sjogren's syndrome, psychotic illness, diabetes, sleep disordered breathing, endometriosis,)

Obstetric history (previous Caesarean section, vaginal bleeding in pregnancy, antenatal care attendance, abruption, previous stillbirth, preterm birth, SGA, IVF)

Obstetric cholestasis

Cigarette smoking, smokeless tobacco and second hand smoking exposure

Caffeine and alcohol intake

Aboriginal status

Ethnicity

Perceived reduced fetal movements

Ultrasound markers

Uterine artery Doppler (UtAD)

Cerebroplacental ratio (CPR)

Middle cerebral artery (MCA)

Fetal nuchal translucency (NT)

Any suboptimal fetal growth

Fetal echogenic bowel

Male fetus

Biochemical markers

Prothrombotic markers

Factor $\mathrm{V}$ Leiden gene mutation

Anticardiolipin Antibodies (ACA)

Lupus anticoagulant (LA)

AB2G1

Protein $S$ deficiency

Activated Protein C Resistance

Prothrombin G20210A mutation

MTHFR C677T mutation

Protein C deficiency

Markers of fetoplacental unit endocrine dysfunction

Human chorionic gonadotrophin (HCG)

Human placental lactogen (hPL)

Alpha-Fetoprotein (AFP)

Pregnancy-associated plasma protein A (PAPP-A)

Estriol

PLGF

Other markers

Thyroid stimulating hormone (TSH)

Haemoglobin $<10$

Serum uric acid

Vitamin D

Proteinuria

Bile acids

Combination of markers

Combinations of first trimester biomarkers

$(A F P+h C G)(P A P P-A+h C G)(A F P+h C G+u E)(A F P+U E)(h C G+u E)$ 
Table 2. Assessment of the evidence supporting the association of individual variables with stillbirth and perinatal mortality - highly convincing, highly suggestive and suggestive evidence.

\begin{tabular}{|c|c|c|c|c|c|c|c|c|c|}
\hline Reference & Variable & Level of comparison & $\begin{array}{l}\text { Sample size } \\
\text { (cases) }\end{array}$ & $\begin{array}{l}\text { Sample size } \\
\text { (events) }\end{array}$ & Heterogeneity & $\begin{array}{c}\text { Small study } \\
\text { effect }\end{array}$ & $\begin{array}{l}\text { Effect size } \\
\text { measure }\end{array}$ & $\begin{array}{c}\text { Random effects } \\
\text { summary effect size }\end{array}$ & $\begin{array}{l}\text { Significance } \\
\text { threshold }\end{array}$ \\
\hline \multicolumn{10}{|c|}{ Highly convincing evidence (>1000 events, $<50 \%$ heterogeneity, no evidence small study effect, $p<0.000001$ ) } \\
\hline Flenady 2011 & Parity & Nulliparity v Multiparity & 24977570 & 74457 & $0.00 \%$ & None & ES & $1.42(1.33-1.51)$ & $<0.000001^{*}$ \\
\hline Flenady 2011 & $\begin{array}{l}\text { Pre-existing } \\
\text { hypertension }\end{array}$ & Affected $v$ unaffected & 23002442 & 66240 & $45.30 \%$ & None & ES & $2.58(2.13-3.13)$ & $<0.000001^{*}$ \\
\hline Liu 2016 & BMI & $>30 v<25$ & 1392799 & NR & $38.40 \%$ & None & OR & $1.81(1.69-1.93)$ & $<0.000001^{*}$ \\
\hline \multicolumn{10}{|c|}{ Highly suggestive evidence (>1000 cases, $p<0.000001$ ) } \\
\hline Lean 2017 & Advanced & $>35 v<35$ years old & 44723207 & 185384 & $95.60 \%$ & None & OR & $1.75(1.62-1.89)$ & $<0.000001^{*}$ \\
\hline Mondal 2014 & Fetal sex & Male $v$ female & 30840461 & 183742 & $71.90 \%$ & None & $\mathrm{RR}$ & $1.1(1.07-1.13)$ & $<0.000001^{*}$ \\
\hline Malacova & Previous SGA & Affected $v$ unaffected & 1602682 & 6782 & $72.40 \%$ & None & OR & $1.85(1.42-2.4)$ & $<0.000001^{*}$ \\
\hline Mugî́ 2019 & Ethnicity & Black v White at 37 & 3081859 & 1053 & 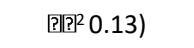 & None & OR & $1.9(1.66-2.17)$ & $<0.000001^{*}$ \\
\hline Oteng-Ntim & Sickle cell disease & Affected $v$ unaffected & 26212461 & $N R$ & (四 0.050$)$ & NR & $\mathrm{RR}$ & $3.99(2.63-6.04)$ & $<0.000001^{*}$ \\
\hline Pineles 2016 & Smoking & Any $v$ none & 23442770 & NR & $67 \%$ & Present & $\mathrm{RR}$ & $1.46(1.38-1.54)$ & $<0.000001^{*}$ \\
\hline Shah 2011 & Aboriginal women & $\begin{array}{l}\text { Aboriginal v non- } \\
\text { Aboriginal }\end{array}$ & 5552134 & NR & High & Present & OR & $1.68(1.49-1.89)$ & $<0.000001^{*}$ \\
\hline \multicolumn{10}{|c|}{ Suggestive evidence (>1000 cases, $p<0.001$ ) } \\
\hline $\begin{array}{c}\text { Malacova } \\
2018\end{array}$ & $\begin{array}{c}\text { Previous preterm } \\
\text { SGA }\end{array}$ & Affected $v$ unaffected & 1309183 & 5250 & $88 \%$ & None & OR & $3.15(1.89-5.25)$ & $0.00001^{*}$ \\
\hline Keag 2018 & Previous CS & Affected $v$ unaffected & 703562 & 2401 & $34 \%$ & None & OR & $1.27(1.15-1.4)$ & $<0.00001$ \\
\hline Yu 2017 & Diabetes & Pre-gestational v none & 24906160 & NR & $48 \%$ & None & OR & 3.52 (3.19-3.88) & $<0.00001$ \\
\hline Oldereid & Paternal age & $<35 v>35$ & 5319012 & $N R$ & $74.70 \%$ & None & OR & $1.19(1.1-1.3)$ & $<0.0001$ \\
\hline Wê̄b̂ 2005 & Psychotic illness & Affected $v$ unaffected & 1688137 & 6012 & $\chi 2=6.35, d f=5$ & Not assessed & OR & $1.89(1.36-2.62)$ & $0.0002^{*}$ \\
\hline Lamont 2015 & Previous stillbirth & Affected $v$ unaffected & 3412079 & 14283 & $8 \hat{2} \hat{\%}^{-}$ & Present & OR & $4.83(3.77-6.18)$ & $<0.001$ \\
\hline Vos 2014 & Social deprivation & $\begin{array}{l}\text { Most deprived quintile v } \\
\text { least deprived }\end{array}$ & 1857057 & $N R$ & $0 \%$ & NR & OR & $1.33(1.21-1.45)$ & $<0.001$ \\
\hline
\end{tabular}


Figure 1. PRISMA flow chart

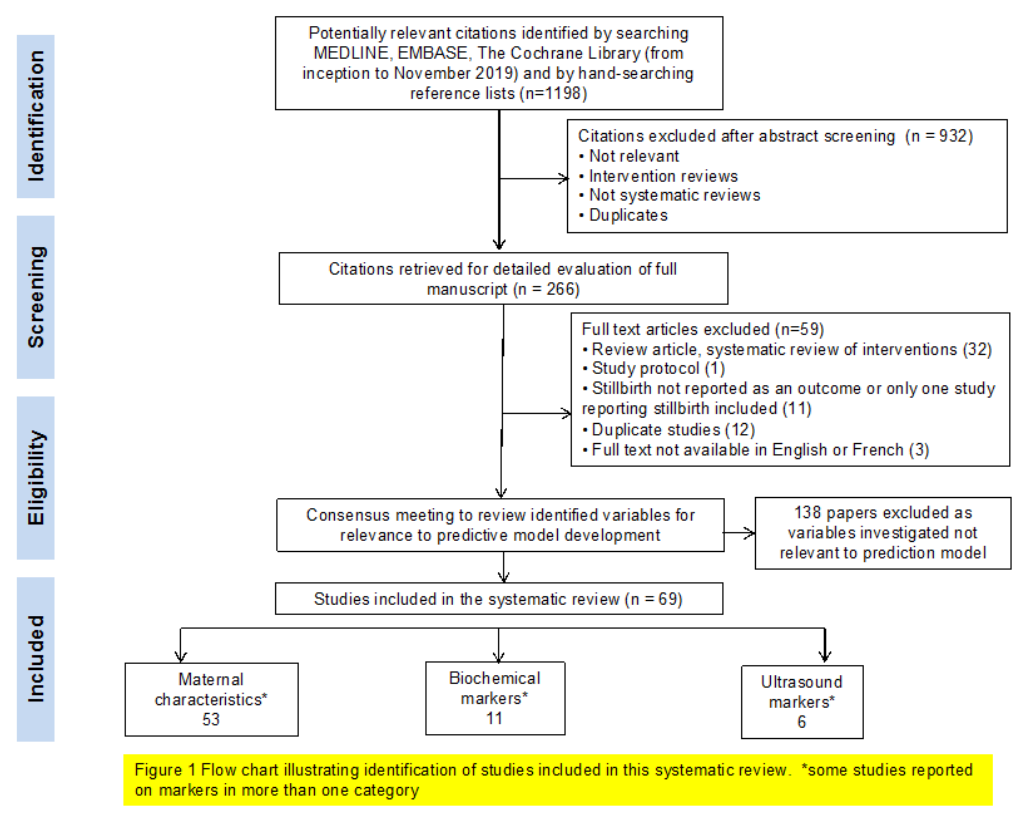

Figure 2a. Methodological quality of included reviews

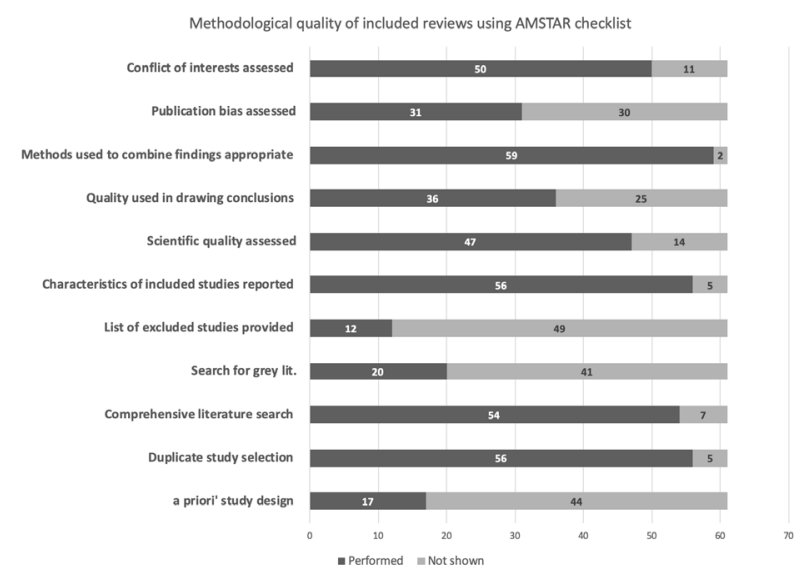

This article is protected by copyright. All rights reserved
Figure $\mathbf{2 b}$. Risk of bias relating to QUIPS domains in the included studies

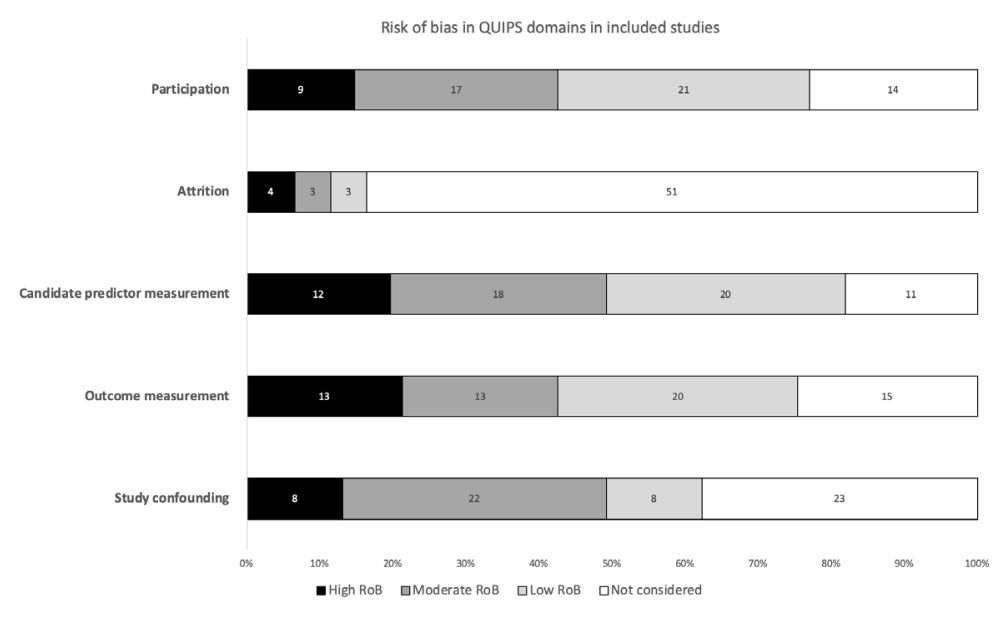

Figure 3. Summary of characteristics of included studies

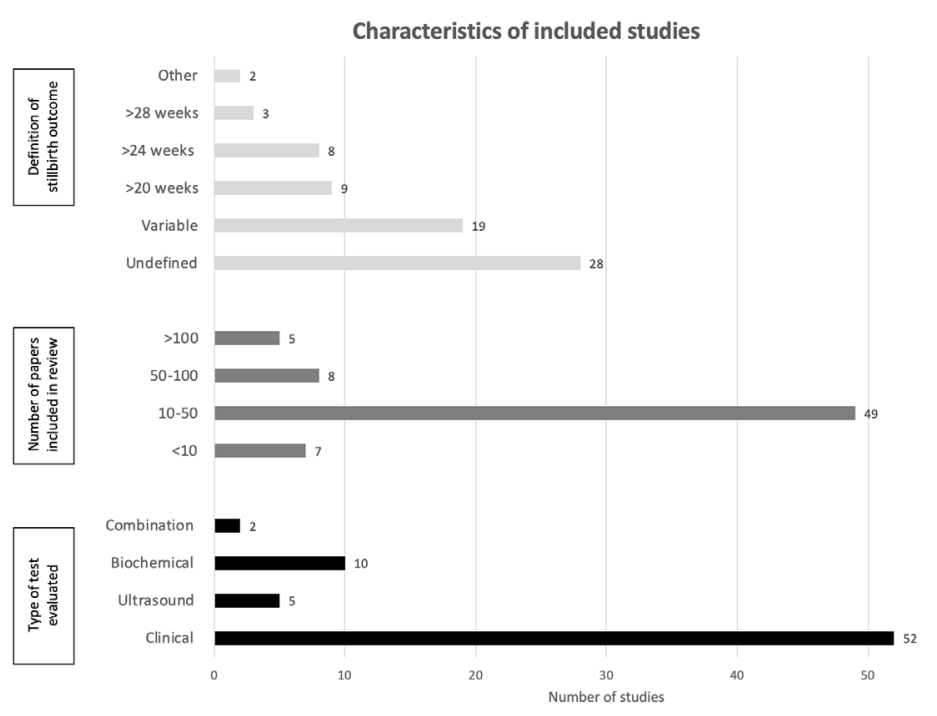


